\title{
SOME RESULTS ON GALOIS REPRESENTATIONS ATTACHED TO MOTIVES OF $G L_{2}$-TYPE
}

\author{
Chuangxun Cheng
}

\begin{abstract}
In this paper, we study the structure of $\operatorname{End}(M)$ where $M$ is a motive of $G L_{2}$-type over a totally real field. Under some standard conjectures for motives, we then prove some results about Galois representations attached to such motives.
\end{abstract}

Keywords: Galois representation, motives of $G L_{2}$-type.

\section{Introduction}

In this paper, a motive always means a motive for absolute Hodge cycles. All the terminology about motives used in this paper will be introduced in Section 2. (See Section 2 for some basic properties and [6] for details.)

Let $K$ be a number field. An abelian variety $A$ over $K$ is of $G L_{2}$-type if the $\mathbb{Q}$-algebra $\operatorname{End}_{K}(A) \otimes_{\mathbb{Z}} \mathbb{Q}$ is a number field of degree $\operatorname{dim}(A)$ over $\mathbb{Q}$. In [7], the author investigates abelian varieties of $G L_{2}$-type and proves that all abelian varieties of $G L_{2}$-type over $\mathbb{Q}$ are modular under Serre's conjecture on $\bmod p$ Galois representations of $G_{\mathbb{Q}}$. In [12], the author proves a similar modularity result for motives of $G L_{2}$-type over $\mathbb{Q}$. In this paper, we continue the work in [12] and prove several results on Galois representations attached to motives of $G L_{2}$-type over totally real fields. As in [12], some of the arguments in this paper are identical to those in [7] and [8].

Let $M$ be a motive over a number field $K$. We say that $M$ is of $G L_{2}$-type if (1) $M$ is pure of weight $w$ with Hodge type $\left(p_{\sigma}, q_{\sigma}\right)+\left(q_{\sigma}, p_{\sigma}\right)\left(p_{\sigma}>q_{\sigma}\right)$ with respect to $\sigma: K \rightarrow \mathbb{C}$, and $w$ is equal to the dimension of the underlying variety of $M$ if $w$ is even; (2) there exists a number field $E$ such that $E \hookrightarrow \operatorname{End}_{K}(M) \otimes_{\mathbb{Z}} \mathbb{Q}$ and $[E: \mathbb{Q}]=\frac{1}{2} \operatorname{rank}(M)$. By functoriality, $E$ acts on $H_{B}(M)$ which is a $\mathbb{Q}$-vector space of dimension $\operatorname{rank}(M)$.

In this paper, we always assume that $K$ is a totally real field. We have the following theorem.

2010 Mathematics Subject Classification: primary: 11G25 
Theorem 1.1. Let $M$ be a motive over $K$ of $G L_{2}$-type. Then $M_{/ K}$ is simple if and only if $E=\operatorname{End}_{K}(M) \otimes \mathbb{Q}$.

Let $N$ be a motive over $\overline{\mathbb{Q}}$ which is pure of weight. We say that $N$ has complex multiplication if it is the product of motives $N_{i}$ such that $\operatorname{End}_{\overline{\mathbb{Q}}}\left(N_{i}\right) \otimes_{\mathbb{Z}} \mathbb{Q}$ is a number field of degree $\operatorname{rank}\left(N_{i}\right)$ over $\mathbb{Q}$. of [12].

We have the following theorem. The case when $K=\mathbb{Q}$ is proved in Theorem 4.3

Theorem 1.2. Let $M$ be a simple motive over $K$ of $G L_{2}$-type such that $M_{\overline{\mathbb{Q}}}=$ $M \times_{K} \overline{\mathbb{Q}}$ does not contain submotives with complex multiplication. Let $E \hookrightarrow$ $\operatorname{End}_{K}(M) \otimes_{\mathbb{Z}} \mathbb{Q}$ with $[E: \mathbb{Q}]=\frac{1}{2} \operatorname{rank}(M)$. Then $\mathfrak{D}:=\operatorname{End}_{\overline{\mathbb{Q}}}(M) \otimes_{\mathbb{Z}} \mathbb{Q}$ is a central simple algebra over a totally real field $L \subset E$, the Schur index of $\mathfrak{D}$ is 1 or 2 , and $\mathfrak{D}$ contains $E$ as a maximal subfield.

Let $M$ be a simple motive of $G L_{2}$-type over $K$ which is pure of weight $w$. Assume that $E=\operatorname{End}_{K}(M) \otimes_{\mathbb{Z}} \mathbb{Q}$. Since $M$ has a polarization (see Section 6 of [4]), we see that $E$ is a totally real or a CM field. Let $V_{l}=H_{l}(M)$ be the $l$-adic realization of $M$ for each prime number $l$ and $E_{\lambda}$ be the completion of $E$ at a finite place $\lambda \mid l$ of $E$. By functoriality, $E$ acts on $V_{l}$ which is a $\mathbb{Q}_{l}$-vector space of $\operatorname{dimension} \operatorname{rank}(M)$. Then $V_{l}$ is free of rank 2 over $E \otimes \mathbb{Q}_{l}, V_{\lambda}:=V_{l} \otimes_{E \otimes \mathbb{Q}_{l}} E_{\lambda}$ is a vector space of dimension 2 over $E_{\lambda}$. Therefore, we get a family of rank 2 Galois representations:

$$
\rho_{\lambda}: G_{K} \rightarrow G L_{E \otimes \mathbb{Q}_{l}}\left(V_{l}\right) \cong G L_{2}\left(E \otimes \mathbb{Q}_{l}\right) \rightarrow G L_{2}\left(E_{\lambda}\right),
$$

where $G_{K}=\operatorname{Gal}(\overline{\mathbb{Q}} / K)$ is the absolute Galois group of $K$.

For each finite place $\lambda$ of $E$, let $\delta_{\lambda}=\operatorname{det}\left(\rho_{\lambda}\right): G_{K} \rightarrow E_{\lambda}^{\times}$. Let $\chi_{l}$ be the $l$-adic cyclotomic character. Fix embeddings $E \hookrightarrow \mathbb{C}$ and $E_{\lambda} \hookrightarrow \mathbb{C}$. Then we have the following theorem. The corresponding results for motives over $\mathbb{Q}$ are proved in Section 5 of [12].

\section{Theorem 1.3.}

(1) Let $M$ be a simple motive of $G L_{2}$-type over $K$ which is pure of weight $w$. Then there is a character of finite order $\epsilon: G_{K} \rightarrow E_{\lambda}^{\times}$such that $\delta_{\lambda}=\epsilon \chi_{l}^{w}$ for each $\lambda$. Furthermore, this character is unramified at each prime which is a prime of good reduction for $M$.

(2) For each $\lambda, \delta_{\lambda}$ is totally odd in the sense that $\delta_{\lambda}(c)=-1$ for any complex conjugation $c \in G_{K}$.

We prove the following theorem. These results in the case $K=\mathbb{Q}$ are mentioned in Section 5 of [12]. The abelian varieties analogue is proved in [7]. Note that if $K=\mathbb{Q}$, Serre's conjecture is now a theorem, thus $\rho_{\lambda}$ is modular and the following results follow immediately from the properties of Galois representations attached to modular forms. 
Theorem 1.4. Under Tate conjecture (see Section 2 for the statement), we have the following results.

(1) For each prime $\mathfrak{p}$ of good reduction of $M, a_{\mathfrak{p}}=\epsilon\left(\right.$ Frob $\left._{\mathfrak{p}}\right) \bar{a}_{\mathfrak{p}}$, where Frob $_{\mathfrak{p}}$ is arithmetic Frobenius, $a_{\mathfrak{p}}=\operatorname{Trace}\left(\rho_{\lambda}\left(\right.\right.$ Frob $\left.\left._{\mathfrak{p}}\right)\right)$, and ${ }^{-}$means the complex conjugation.

(2) Let $S$ be a finite set of finite primes at which $M$ has bad reduction. Then $E=\mathbb{Q}\left(a_{\mathfrak{p}} \mid \mathfrak{p} \notin S\right)$.

(3) Let $F$ be the subfield of E generated by the numbers $a_{\mathfrak{p}}^{2} / \epsilon\left(\right.$ Frob $\left._{\mathfrak{p}}\right)$ with $\mathfrak{p} \notin S$, then $F$ is totally real and the extension $E / F$ is abelian.

(4) The field $L$ in Theorem 1.2 is the same as the field $F$ in (3). In particular, $E$ is an abelian extension of $L$.

\section{Motives for absolute Hodge cycles}

In this section, we review the construction of the category of motives for absolute Hodge cycles. Let $X$ be a smooth projective variety over a subfield $K$ of $\mathbb{C}$. We consider three Weil cohomologies $H_{d R}, H_{B, \sigma}$, and $H_{l}$. (They are de Rham cohomology, Betti cohomology with respect to $\sigma: K \hookrightarrow \mathbb{C}$, and $l$-adic etale cohomology.) These cohomologies are furnished with cycle maps:

$$
c l^{r}: C H^{r}(X) \rightarrow H_{?}^{2 r}(X)(r),
$$

where $C H^{r}(X)$ is the abelian group of algebraic cycles of codimension $r$ on $X$ modulo rational equivalence, and $?=d R,(B, \sigma)$, or $l$. Define

$$
H_{\text {total }}^{2 r}(X)(r):=H_{d R}^{2 r}(X)(r) \times \prod_{l} H_{l}^{2 r}(X)(r) \times \prod_{\sigma} H_{B, \sigma}^{2 r}(X, \mathbb{Z}) \otimes \mathbb{Z}(r) .
$$

Let $I_{\sigma}: H_{B, \sigma}(X) \otimes_{\mathbb{Q}} \mathbb{C} \sim{ }_{\rightarrow} H_{d R}(X) \otimes_{K, \sigma} \mathbb{C}$ and $I_{l, \sigma}: H_{B, \sigma}(X) \otimes_{\mathbb{Q}} \mathbb{Q}_{l} \stackrel{\sim}{\rightarrow} H_{l}(X)$ be the comparison isomorphisms. Then write $I_{\sigma, \mathbb{Z}}\left(\operatorname{resp} . I_{l, \sigma, \mathbb{Z}}\right)$ for the composition of the natural inclusion $H_{B, \sigma}^{2 r}(X, \mathbb{Z}) \otimes \mathbb{Z}(r) \hookrightarrow H_{B, \sigma}(X)(r)$ with $I_{\sigma}$ (resp. $I_{l, \sigma}$ ).

Definition 2.1. An absolute Hodge cycle $x$ of codimension $r$ on $X$ is an element of the finitely generated $\mathbb{Z}$-module:

$$
C_{A H}^{r}(X):=\left\{\left(x_{d R}, x_{l}, x_{\sigma}\right)_{l, \sigma} \in H_{\text {total }}^{2 r}(X)(r) \mid I_{\sigma, \mathbb{Z}}\left(x_{\sigma}\right)=x_{d R}, I_{l, \sigma, \mathbb{Z}}\left(x_{\sigma}\right)=x_{l}\right\} .
$$

The cup product of each cohomology theory induces the ring structure on $C_{A H}^{2 \operatorname{dim}(X)}(X \times X)$ for a smooth projective variety $X$.

The category $\mathcal{M}_{A H}$ of motives for absolute Hodge cycles is defined as follows. The objects of $\mathcal{M}_{A H}$ are triples $(X, p, m)$, where $X$ is a smooth projective variety, $p \in C_{A H}^{2 \operatorname{dim}(X)}(X \times X) \otimes \mathbb{Q}$ is a projector, and $m$ is an integer. For two objects $M=(X, p, m)$ and $N=(Y, q, n)$ of $\mathcal{M}_{A H}$, the morphism set is defined by

$$
\operatorname{Hom}(M, N):=p \circ \operatorname{Corr}_{A H}^{-m+n}(X, Y) \circ q,
$$

where $\operatorname{Corr}_{A H}^{r}(X, Y)=\prod_{i=1}^{n} C_{A H}^{r+\operatorname{dim}\left(X_{i}\right)}\left(X_{i} \times Y\right) \otimes \mathbb{Q}$ if $X$ has irreducible components $X_{1}, \cdots, X_{n}$. 
Remark 2.2. There are several advantages to work with motives for absolute Hodge cycles. First, the category of motives for absolute Hodge cycles by extending the coefficients to $\mathbb{Q}$ is a semi-simple $\mathbb{Q}$-linear abelian category. (See for example [6] and Section 6 of [4].) Then we can control the decomposition of motives using their endomorphism algebras and consider the image of a morphism between motives. See [12] for applications of this fact. Second, there is a well defined theory of polarizations for motives for absolute Hodge cycles. (See Section 6 of [4].) Therefore, we may generalize certain arguments in the case of abelian varieties to the case of motives.

In the rest of this section, we recall some definitions related to motives for absolute Hodge cycles.

Dual motives. Let $M=(X, p, m)$ be a pure motive, we define the dual motive $M^{\vee}=\left(X, p^{t}, \operatorname{dim}(X)-m\right)$, where $p^{t}$ means the transpose of $p$.

The motive $\mathbb{L}=\left(\mathbb{P}^{1}, \pi_{2}, 0\right), \pi_{2}=\mathbb{P}^{1} \times\{p t\}$, is the so-called Lefschetz motive and $\mathbb{Q}(1):=\mathbb{L}^{\vee}=\left(\mathbb{P}^{1}, \pi_{0}, 1\right), \pi_{0}=\{p t\} \times \mathbb{P}^{1}$, is the so-called Tate motive. For $M=(X, p, m)$, we have isomorphism $M \cong(X, p, 0) \otimes \mathbb{Q}(m)$.

Realizations of motives. For each Weil cohomology theory $H_{\text {? }}$, we define the realizations of a motive $M=(X, p, 0)$ by

$$
H_{?}^{*}(M)=\oplus_{i=0}^{2 \operatorname{dim}(X)} H_{?}^{i}(M),
$$

where $H_{?}^{i}(M)=\operatorname{Im}\left(p_{*}: H_{?}^{i}(X) \rightarrow H_{?}^{i}(X)\right)$. Here $p_{*}$ is the composite map of the following maps

$$
H_{?}^{i}(X) \stackrel{p_{1}^{*}}{\longrightarrow} H_{?}^{i}(X \times X) \stackrel{\cup \gamma(p)}{\longrightarrow} H_{?}^{i+2 \operatorname{dim}(X)}(X \times X) \stackrel{p_{2 *}}{\longrightarrow} H_{?}^{i}(X),
$$

where $p_{j}: X \times X \rightarrow X$ is the $j$-th projection, $\gamma: \operatorname{Corr}^{0}(X, X) \rightarrow H_{?}^{2 \operatorname{dim}(X)}(X)$ is the cycle map, and $p_{2 *}$ is the Gysin homomorphism.

Definition 2.3. Let $M=(X, p, m)$ be a motive. If $H_{?}^{*}(M) \subset H_{?}^{i}(X)(m)$ for each Weil cohomology $H_{?}=H_{B, \sigma}, H_{d R}, H_{\text {et }}$, then we say that $M$ is pure of weight $w=i-2 m$. Note that if $M$ is pure of weight $w$, then $M^{\vee}=M(w)$.

We have Hodge decomposition $H_{B, \sigma}^{i}(X)(m)=\oplus_{p+q=i} H_{B, \sigma}^{p, q}(X)(m)$. We say $M$ is of Hodge type $\left(p_{\sigma}, q_{\sigma}\right)+\left(q_{\sigma}, p_{\sigma}\right)$ if $H_{B, \sigma}^{*}(M) \subset H_{B, \sigma}^{p_{\sigma}, q_{\sigma}}(X)(m) \oplus H_{B, \sigma}^{q_{\sigma}, p_{\sigma}}(X)(m)$.

If $M=(X, p, m)$ is pure of weight $i-2 m$ and $M$ is defined over a number field $K$ (i.e., $X$ and $p$ are both defined over $K$ ), then the realizations of $M$ can be explicitly described. See Section 2 of [12] and Section 4 of [6] for details.

The rank of a motive. We have comparison isomorphisms between realizations of motives:

$$
I_{\sigma}: H_{B, \sigma}(M) \otimes_{\mathbb{Q}} \mathbb{C} \stackrel{\sim}{\rightarrow} H_{d R}(M) \otimes_{K, \sigma} \mathbb{C}
$$

and

$$
I_{l, \sigma}: H_{B, \sigma}(M) \otimes_{\mathbb{Q}} \mathbb{Q}_{l} \stackrel{\sim}{\longrightarrow} H_{l}(M) .
$$

We define the rank of $M$ to be $\operatorname{dim}_{\mathbb{Q}} H_{B, \sigma}(M)$. By the comparison theorem, $\operatorname{rank}(M)=\operatorname{dim}_{\mathbb{Q}_{l}}\left(H_{l}(M)\right)=\operatorname{dim}_{K}\left(H_{d R}(M)\right)$. 
Field of definition of an endomorphism. As in [12], we define the field of definition of absolute Hodge cycles as follows. For a motive $M$ over $K$, we say $\phi \in \operatorname{End}(M)$ is defined over a field extension $L$ of $K$ if $(\phi)_{l}$ is member of $H_{l}(M)^{\operatorname{Gal}(\bar{K} / L)}$ for all prime $l$. We write $\operatorname{End}_{L}(M)$ for the ring consisting of endomorphisms of $M$ defined over $L$.

Tate conjecture. We recall the Tate conjecture as stated in Section 5 of [12]. For more details, see the references cited in [12].

Conjecture 2.4 (Tate conjecture). Let $M$ and $N$ be motives over a number field $K$ for absolute Hodge cycles which have same weight. Let $B$ be a positive number such that $H_{e t}\left(M, \mathbb{Z}_{l}\right)$ and $H_{e t}\left(N, \mathbb{Z}_{l}\right)$ are free $\mathbb{Z}_{l}$-modules for any prime number $l \geqslant B$. Let $\rho_{l}: G_{K} \rightarrow G L_{\mathbb{Z}_{l}}\left(H_{e t}\left(M, \mathbb{Z}_{l}\right)\right)$ be the l-adic representation attached to $M$. Then we have the following.

(1) $H_{e t}\left(M, \mathbb{Z}_{l}\right)$ is a semi-simple $G_{K}$-module.

(2) The natural map

$$
\alpha_{K}: \operatorname{Hom}_{K}(N, M) \otimes_{\mathbb{Z}} \mathbb{Z}_{l} \rightarrow \operatorname{Hom}_{G_{K}}\left(H_{e t}\left(N, \mathbb{Z}_{l}\right), H_{e t}\left(M, \mathbb{Z}_{l}\right)\right)
$$

is an isomorphism.

(3) For almost all $l \geqslant B$, the subalgebra $\mathbb{Z}_{l}\left[\rho_{l}\left(G_{K}\right)\right]$ of $\operatorname{End}_{\mathbb{Z}_{l}}\left(H_{e t}\left(M, \mathbb{Z}_{l}\right)\right)$ is the full commutator of the image of the natural map $\beta_{K}: \operatorname{End}_{K}(M) \rightarrow$ $\operatorname{End}_{\mathbb{Z}_{l}}\left(H_{e t}\left(M, \mathbb{Z}_{l}\right)\right)$.

(4) Let $\bar{\rho}_{l}$ be the reduction of $\rho_{l} \bmod l$ for $l \geqslant B$. Then for almost all $l \geqslant$ $B$, the subalgebra $\mathbb{F}_{l}\left[\bar{\rho}_{l}\left(G_{K}\right)\right]$ of $\operatorname{End}_{\mathbb{F}_{l}}\left(H_{e t}\left(M, \mathbb{Z}_{l}\right) / l H_{e t}\left(M, \mathbb{Z}_{l}\right)\right)$ is the full commutator of the image of the natural map $\bar{\beta}_{K}: \operatorname{End}_{K}(M) \otimes \mathbb{Z} / l \mathbb{Z} \rightarrow$ $\operatorname{End}_{\mathbb{F}_{l}}\left(H_{e t}\left(M, \mathbb{Z}_{l}\right) / l H_{e t}\left(M, \mathbb{Z}_{l}\right)\right)$.

(5) $\operatorname{End}_{\mathbb{F}_{l}}\left(H_{e t}\left(M, \mathbb{Z}_{l}\right) / l H_{e t}\left(M, \mathbb{Z}_{l}\right)\right)$ is a semi-simple $G_{K}$-module.

\section{Endomorphisms of $M$}

\subsection{Proof of Theorem 1.1 and Theorem 1.2}

We prove Theorem 1.1 and 1.2 in this subsection. The strategy is essentially the same as in the case $K=\mathbb{Q}$.

Proof of theorem 1.1. Let $\mathfrak{X}=\operatorname{End}_{K}(M) \otimes \mathbb{Q}$. By assumption, we have embedding $E \hookrightarrow \mathfrak{X}$. Let $D$ be the commutant of $E$ in $\mathfrak{X}$. First, we show that $D$ is a field.

Let $\phi \in D \backslash\{0\}$ and let $N=\phi(M)$. Certainly, $E$ acts on $N$. $\phi$ induces an endomorphism of $H_{d R}(M)$ which preserves the filtration of $H_{d R}(M)$. Since $K$ is totally real, by Lemma 5.1 .1 of [5], $H_{d R}(M)$ (resp. $\left.H_{d R}(N)\right)$ has a filtration $F(M)=F i l^{[w / 2]} H_{d R}(M)$ (resp. $\left.F(N)\right)$ which is a free $E \otimes K$-module of rank one (resp. of $\left.\operatorname{rank} \frac{\operatorname{rank}(N)}{2}\right)$. Since

$$
[E: \mathbb{Q}] \leqslant \frac{\operatorname{rank}_{K}\left(H_{d R}(N)\right)}{2} \leqslant \frac{\operatorname{rank}_{K}\left(H_{d R}(M)\right)}{2}=[E: \mathbb{Q}]
$$

we have $\operatorname{rank}(M)=\operatorname{rank}(N)$ and $\phi$ is an isogeny. $D$ is a division algebra over $\mathbb{Q}$ such that $D \supset E$. 
Because endomorphisms of $M$ preserve the filtration of $H_{d R}(M), F(M)$ can be viewed as a $D$-vector space. Then $\operatorname{dim}_{K} D \otimes K \mid \operatorname{rank}_{K}(F(M))=[E: \mathbb{Q}][K: \mathbb{Q}]$. Therefore $[D: \mathbb{Q}] \mid[E: \mathbb{Q}]$. So $E=D$. In particular, the center $F$ of $\mathfrak{X}$ is a subfield of $D=E$.

Assume that $M \sim_{K} \prod_{i} N_{i}^{n_{i}}$, where $N_{i}$ is a simple motive over $K$. Then $\mathfrak{X}=$ $\prod_{i} M_{n_{i}}\left(D_{i}\right)$, where $D_{i}=\operatorname{End}_{K}\left(N_{i}\right) \otimes \mathbb{Q}$ is a division algebra over $F$. Since $E \hookrightarrow \mathfrak{X}$, and the commutant of $E$ in $\mathfrak{X}$ is $E$ itself, we may assume that $M \sim_{K} N_{i}^{n_{i}}$ for one $i$. Let $t_{i}=\sqrt{\operatorname{dim}_{\mathbb{Q}} D_{i}}$, then $[E: F]=n_{i} t_{i}$. As before, $F\left(N_{i}\right)$ is a vector space over $D_{i}$, we have $\operatorname{dim}_{\mathbb{Q}} D_{i} \mid \operatorname{rank}_{K}\left(F\left(N_{i}\right)\right)$. Therefore $n_{i} \cdot \operatorname{dim}_{\mathbb{Q}} D_{i} \mid n_{i} \cdot \operatorname{rank}_{K}\left(F\left(N_{i}\right)\right)$. Note that $n_{i} \cdot \operatorname{dim}_{\mathbb{Q}} D_{i}=n_{i} t_{i}^{2}[F: \mathbb{Q}], n_{i} \cdot \operatorname{rank}_{K}\left(F\left(N_{i}\right)\right)=\operatorname{rank}_{K}(F(M))=$ $[E: \mathbb{Q}]=t_{i} n_{i}[F: \mathbb{Q}]$, so $t_{i}=1, F=D_{i}$. The theorem follows.

Proof of theorem 1.2. Since $M_{/ K}$ is simple, we have $E=\operatorname{End}_{K}(M) \otimes \mathbb{Q}$. First, we assume that $M$ is $\overline{\mathbb{Q}}$ simple. Let $D$ be the commutant of $E$ in $\mathfrak{D}=\operatorname{End}_{\overline{\mathbb{Q}}}(M) \otimes$ $\mathbb{Q}$. By the same argument as in the proof of Theorem 1.1, $\operatorname{dim}_{\mathbb{Q}} D \mid[E: \mathbb{Q}]$ and $D=E$. Let $F=Z(\mathfrak{D})$ be the center of $\mathfrak{D}$. Assume that $\mathfrak{D}$ has Schur index $t$, then we have $[E: F]=t,[\mathfrak{D}: F]=t^{2}$. Note that $\operatorname{rank}(M)=2[E: \mathbb{Q}]=$ $2 t[F: \mathbb{Q}]$. Since $H_{B}(M)$ is vector space over $\mathfrak{D}$, we have $t^{2}[F: \mathbb{Q}]=\operatorname{rank}_{\mathbb{Q}}(\mathfrak{D}) \mid$ $\operatorname{rank}_{\mathbb{Q}}\left(H_{B}(M)\right)=2 t[F: \mathbb{Q}]$. Therefore $t=1$ or 2 .

We still have to prove that $F$ is totally real. This is a statement about motives over $\overline{\mathbb{Q}}$ (it has nothing to do with the field of definition of $M$ ). It follows from the argument in the proof of Theorem 4.3 of [12].

Now assume that $M \sim_{\overline{\mathbb{Q}}} \prod_{i} N_{i}^{n_{i}}$, where $N_{i}$ are $\overline{\mathbb{Q}}$ simple. Then

$$
\operatorname{End}_{\overline{\mathbb{Q}}}(M)=\prod_{i} M_{n_{i}}\left(\operatorname{End}_{\overline{\mathbb{Q}}}\left(N_{i}\right)\right) .
$$

Because $E$ is a $\mathbb{Q}$ subalgebra of $E n d_{\overline{\mathbb{Q}}}(M) \otimes \mathbb{Q}$, we must have $E \hookrightarrow M_{n_{i}}\left(\operatorname{End}_{\overline{\mathbb{Q}}}\left(N_{i}\right)\right)$ for each $i$. Then

$$
\frac{\operatorname{rank}(M)}{2}=[E: \mathbb{Q}] \mid \frac{n_{i}}{2} \operatorname{rank}\left(N_{i}\right) \leqslant \sum_{i} \frac{n_{i}}{2} \operatorname{rank}\left(N_{i}\right)=\frac{\operatorname{rank}(M)}{2} .
$$

We must have $M \sim_{\overline{\mathbb{Q}}} N^{n}$. Then $\operatorname{End}_{\overline{\mathbb{Q}}}(M)=M_{n}\left(\operatorname{End}_{\overline{\mathbb{Q}}}(N)\right)$, the statements hold.

\section{2. $K$-motives}

Let $M$ be a motive over $\overline{\mathbb{Q}}, K$ be a number field. We say $M$ is a $K$-motive if (1) $M$ is pure of weight $w$ with Hodge type $\left(p_{\sigma}, q_{\sigma}\right)+\left(q_{\sigma}, p_{\sigma}\right)\left(p_{\sigma}>q_{\sigma}\right)$, and $w$ is equal to the dimension of the underlying variety of $M$ if $w$ is even; $(2) \operatorname{End}_{\overline{\mathbb{Q}}}(M) \otimes \mathbb{Q}$ is a division algebra over a totally real field $F$ with Schur index $t=1$ or 2 , and $2 t[F: \mathbb{Q}]=\operatorname{rank}(N) ;(3)$ for each $\sigma \in \operatorname{Gal}(\overline{\mathbb{Q}} / K)$, there exists a non-zero isogeny

$$
\mu_{\sigma}: M^{\sigma} \rightarrow M
$$

such that $\mu_{\sigma} \circ \sigma(\phi)=\phi \circ \mu_{\sigma}$ for all $\phi \in \operatorname{End}_{\overline{\mathbb{Q}}}(M) \otimes_{\mathbb{Z}} \mathbb{Q}$. We then have the following proposition. 
Proposition 3.1. Let $N$ be a $\overline{\mathbb{Q}}$ simple factor of a motive $M$ of $G L_{2}$-type over $K$ which does not contain submotives with complex multiplication. Then $N$ is a K-motive.

Proof. From the proof of Theorem 1.2, we may assume that $M \sim_{\overline{\mathbb{Q}}} N^{n}$, where $N$ is a $\overline{\mathbb{Q}}$ simple motive. We have proved that the endomorphism algebra $\operatorname{End}_{\overline{\mathbb{Q}}}(N) \otimes$ $\mathbb{Q}$ is a division algebra over a totally real field $F$ with Schur index $t=1$, or 2 , and the $\operatorname{rank}$ of $N$ is $\operatorname{rank}(N)=t[F: \mathbb{Q}]$. Then the result follows exactly by the same argument as in the proof of Proposition 6.3 of [12].

\section{Galois representations attached to $M$}

\subsection{Proof of Theorem 1.3 and Theorem 1.4}

Proof of theorem 1.3. The proof is the same as the proof of Lemma 5.1 of [12]. The only difference is that, for statement (2), we have a complex conjugation for each embedding $K \hookrightarrow \overline{\mathbb{C}}$. But the argument in [12] still holds since the complex conjugation induced from $\sigma: K \rightarrow \mathbb{C}$ sends $H^{p_{\sigma}, q_{\sigma}}(M)$ to $H^{q_{\sigma}, p_{\sigma}}(M)$ and $p_{\sigma} \neq q_{\sigma}$.

Proof of theorem 1.4. (1) Let $\sigma: E \hookrightarrow \overline{\mathbb{Q}}_{l}$ be an embedding of fields. Let $\bar{\sigma}$ be the complex conjugation of $\sigma$ defined by $\bar{\sigma}(x)=\sigma(\bar{x})$. (Recall that we have fixed an embedding $E \hookrightarrow \mathbb{C}$.) Let $V_{\sigma}=V_{l} \otimes_{E \otimes \mathbb{Q}_{l}} \overline{\mathbb{Q}}_{l}$, where the tensor product is taken relative to the map $E \otimes \mathbb{Q}_{l} \hookrightarrow \overline{\mathbb{Q}}_{l}$ induced by $\sigma$. Define $V_{\bar{\sigma}}$ similarly. Fix a polarization of $M$ defined over $K$. It gives us a bilinear map

$$
\phi: V_{l} \times V_{l} \rightarrow \mathbb{Q}_{l}(w)
$$

with the following properties:

$$
\begin{aligned}
\phi(e x, y) & =\phi(x, \bar{e} y) & & \forall e \in E, x, y \in V_{l}, \\
\phi(g x, g y) & =g \phi(x, y) & & \forall g \in G_{K} .
\end{aligned}
$$

Here $\bar{e}=e$ if $E$ is totally real, $\bar{e}$ is the complex conjugation of $e$ if $E$ is CM. After extending scalars from $\mathbb{Q}_{l}$ to $\overline{\mathbb{Q}}_{l}$, we find an isomorphism of $\overline{\mathbb{Q}}_{l}\left[G_{K}\right]$-modules

$$
V_{\bar{\sigma}} \cong \operatorname{Hom}\left(V_{\sigma}, \overline{\mathbb{Q}}_{l}(w)\right) \text {. }
$$

By Theorem 1.3, the determinant of $V_{\sigma}$ is a one-dimensional vector space over $\overline{\mathbb{Q}}_{l}$ on which $G_{K}$ acts by the character $\epsilon^{\sigma} \chi_{l}^{w}$. Since $V_{\sigma}$ is of dimension two over $\overline{\mathbb{Q}}_{l}$, this gives us another isomorphism

$$
\operatorname{Hom}\left(V_{\sigma}, \overline{\mathbb{Q}}_{l}\left(\epsilon^{\sigma} \chi_{l}^{w}\right)\right) \cong V_{\sigma} .
$$

By the above two isomorphisms, we have $V_{\sigma} \cong V_{\bar{\sigma}}\left(\epsilon^{\sigma}\right)$. For $\mathfrak{p} \nmid l$ a prime of good reduction, the trace of Frob $_{\mathfrak{p}}$ acting on $V_{\sigma}$ is $\sigma\left(a_{\mathfrak{p}}\right)$, the trace of Frob $b_{\mathfrak{p}}$ acting on $V_{\bar{\sigma}}\left(\epsilon^{\sigma}\right)$ is $\epsilon^{\sigma}\left(\right.$ Frob $\left._{\mathfrak{p}}\right) \bar{\sigma}\left(a_{\mathfrak{p}}\right)=\epsilon^{\sigma}\left(\right.$ Frob $\left._{\mathfrak{p}}\right) \sigma\left(\bar{a}_{\mathfrak{p}}\right)$. The result follows. 
(2) By Tate conjecture, we have $E \otimes \mathbb{Q}_{l}=\left(\operatorname{End}_{K} M\right) \otimes \mathbb{Q}_{l}=\operatorname{End}_{G_{K}} V_{l}$. Let $\bar{V}_{l}=V_{l} \otimes \overline{\mathbb{Q}}_{l}$. Then we have $E n d_{\overline{\mathbb{Q}}_{l}\left[G_{K}\right]} \bar{V}_{l}=E \otimes \overline{\mathbb{Q}}_{l}$. Also, $V_{l}$ is a semi-simple $G_{K}$-module by Tate conjecture, so $\bar{V}_{l}$ is a semi-simple $\overline{\mathbb{Q}}_{l}\left[G_{K}\right]$-module. Define $V_{\sigma}$ as in (1). Then $V_{\sigma}$ are semi-simple $\overline{\mathbb{Q}}_{l}\left[G_{K}\right]$-modules and they are pairwise nonisomorphic. (In fact, they are semi-simple since $\bar{V}_{l}$ is. By the same argument in the proof of Proposition 4.2, the commutator of their product is $\prod_{\sigma} \overline{\mathbb{Q}}_{l}$.) It follows that their traces are pairwise distinct. Let $E^{\prime}=\mathbb{Q}\left(a_{\mathfrak{p}} \mid \mathfrak{p} \notin S\right) \subset E$, we have $\left.\sigma\right|_{E^{\prime}} \neq\left.\tau\right|_{E^{\prime}}$ for any pair $\sigma, \tau: E \hookrightarrow \overline{\mathbb{Q}}_{l}$. So we must have $E=E^{\prime}=\mathbb{Q}\left(a_{\mathfrak{p}} \mid \mathfrak{p} \notin S\right)$.

(3) For $\mathfrak{p} \notin S$, by (1), we have

$$
\frac{a_{\mathfrak{p}}^{2}}{\epsilon\left(\text { Frob }_{\mathfrak{p}}\right)}=\bar{a}_{\mathfrak{p}}^{2} \epsilon\left(\text { Frob }_{\mathfrak{p}}\right)=\frac{\bar{a}_{\mathfrak{p}}^{2}}{\bar{\epsilon}\left(\text { Frob }_{\mathfrak{p}}\right)},
$$

where $^{-}$is complex conjugation. So $F$ is totally real. Then $E$ is contained in the extension of $F$ obtained by adjoining to $F$ the square roots of all $\frac{a_{\mathfrak{p}}^{2}}{\epsilon\left(\text { Frob }_{\mathfrak{p}}\right)}$ and all roots of unity. Therefore $E$ is an abelian extension of $F$.

(4) Choose a prime number $l$ which splits completely in $E$, so that every embedding $E \hookrightarrow \overline{\mathbb{Q}}_{l}$ factors as $E \hookrightarrow \mathbb{Q}_{l} \hookrightarrow \overline{\mathbb{Q}}_{l}$. Choose a finite extension $K^{\prime}$ of $\mathbb{Q}$ which is sufficiently large such that: (1) all endomorphisms of $M$ are defined over $K^{\prime} ;(2) H:=\operatorname{Gal}\left(\overline{\mathbb{Q}} / K^{\prime}\right)$ is contained in the kernel of $\epsilon$. By Tate conjecture, we have $\mathfrak{D} \otimes \mathbb{Q}_{l}=E n d_{\mathbb{Q}_{l}[H]} V_{l}$. The center of $\mathfrak{D} \otimes \mathbb{Q}_{l}$ is $L \otimes \mathbb{Q}_{l}$. The space $V_{l}=H_{l}(M)$ which is free of rank two over $E \otimes \mathbb{Q}_{l}$ decomposes as a product $\prod_{\sigma} V_{\sigma}$, where $\sigma$ runs over the set $\Omega$ of injections $\sigma: E \rightarrow \mathbb{Q}_{l}, V_{\sigma}=V_{l} \otimes_{E \otimes \mathbb{Q}_{l}} \mathbb{Q}_{l}$ with respect to $\sigma: E \hookrightarrow \mathbb{Q}_{l}$. By Tate conjecture, the action of $H$ on $V_{l}$ is semi-simple. So $V_{\sigma}$ is a simple $\mathbb{Q}_{l}[H]$-module because $M$ has no sub motives with complex multiplication. Hence $\operatorname{End}_{H} V_{\sigma}=\mathbb{Q}_{l}$ for all $\sigma$.

Now consider $M$ as a motive defined over $K^{\prime}$. Certainly, we have a Galois representation $r: \operatorname{Gal}\left(\overline{\mathbb{Q}} / K^{\prime}\right) \rightarrow G L_{E}\left(H_{B}(M)\right) \cong G L_{2}(E)$. For each prime $v$ of $K^{\prime}$ which is not over $l$ and at which $M$ has good reduction, let $t_{v} \in E$ be the number $\operatorname{Trace}\left(r\left(\operatorname{Frob}_{v}\right)\right)$. Write $r_{l}$ for the Galois representation

$$
r_{l}: G a l\left(\overline{\mathbb{Q}} / K^{\prime}\right) \rightarrow G L_{E \otimes \mathbb{Q}_{l}}\left(H_{l}(M)\right) \cong G L_{2}\left(E \otimes \mathbb{Q}_{l}\right) \cong \prod_{\sigma} G L\left(V_{\sigma}\right),
$$

we have $\operatorname{Trace}\left(\left.\operatorname{Frob}_{v}\right|_{V_{\sigma}}\right)=\sigma\left(t_{v}\right)$ for each $\sigma$. For two different $\sigma, \tau \in \Omega, V_{\sigma}$ and $V_{\tau}$ are isomorphic as $\mathbb{Q}_{l}[H]$-modules if and only if they have the same trace, i.e., $\sigma\left(t_{v}\right)=\tau\left(t_{v}\right)$ for all $v$, if and only if $\left.\sigma\right|_{J}=\left.\tau\right|_{J}$, where $J$ is the field $\mathbb{Q}\left(t_{v}\right)$. This implies that the center of $\mathfrak{D} \otimes \mathbb{Q}_{l}$ is $J \otimes \mathbb{Q}_{l}$. Thus $L \otimes \mathbb{Q}_{l}=J \otimes \mathbb{Q}_{l}$, which implies that $L=J$.

Assume $\left.\sigma\right|_{J}=\left.\tau\right|_{J}$, so that $V_{\sigma} \cong V_{\tau}$ as representations of $H$. Therefore $\operatorname{Ind}_{H}^{G_{K}}\left(\left.V_{\sigma}\right|_{H}\right) \cong \operatorname{Ind}_{H}^{G_{K}}\left(\left.V_{\tau}\right|_{H}\right)$ as representations of $G_{K}$. Since $V_{\sigma}$ is a simple factor of $\operatorname{Ind}_{H}^{G_{K}}\left(\left.V_{\sigma}\right|_{H}\right)$ and $V_{\tau}$ is a simple factor of $\operatorname{Ind}_{H}^{G_{K}}\left(\left.V_{\tau}\right|_{H}\right)$, we see that there exists a character $\psi: G_{K} \rightarrow \mathbb{Q}_{l}^{\times}$such that $V_{\sigma} \cong V_{\tau} \otimes \psi$ as representations of $G_{K}$. (In fact, we see that $\psi$ factors as $G_{K} \rightarrow \operatorname{Gal}\left(K^{\prime} / K\right) \rightarrow \mathbb{Q}_{l}^{\times}$.) Since both $V_{\sigma}$ and $V_{\tau}$ are unramified at all primes $\mathfrak{p} \nmid l$ and at which $M$ has good reduction, so is $\psi$. Taking the traces of the two representations $V_{\sigma} \cong V_{\tau} \otimes \psi$, we 
have $\sigma\left(a_{\mathfrak{p}}\right)=\psi\left(\right.$ Frob $\left._{\mathfrak{p}}\right) \tau\left(a_{\mathfrak{p}}\right)$ for all such primes. Taking the determinant, we have $\epsilon^{\sigma}=\psi^{2} \cdot \epsilon^{\tau}$. Therefore, $\sigma\left(\frac{a_{\mathfrak{p}}^{2}}{\epsilon\left(\text { Frob }_{\mathfrak{p}}\right)}\right)=\tau\left(\frac{a_{\mathfrak{p}}^{2}}{\epsilon\left(\text { (Frob }_{\mathfrak{p}}\right)}\right)$ for all $\mathfrak{p} \nmid l$ and at which $M$ has good reduction. By Galois theory, we have $\frac{a_{\mathfrak{p}}^{2}}{\epsilon\left(\text { (Frob }_{\mathfrak{p}}\right)} \in J$ for all $\mathfrak{p} \nmid l$ at which $M$ has good reduction. By varying $l$, we see that $\mathbb{Q}\left(\frac{a_{\mathfrak{p}}^{2}}{\epsilon\left(\operatorname{Frob}_{\mathfrak{p}}\right)} \mid \mathfrak{p} \notin S\right) \subset J$.

We have to prove $J \subset \mathbb{Q}\left(\frac{a_{\mathfrak{p}}^{2}}{\epsilon\left(\text { Frob }_{\mathfrak{p}}\right)} \mid \mathfrak{p} \notin S\right)$. If $\sigma\left(\frac{a_{\mathfrak{p}}^{2}}{\epsilon\left(\text { Frob }_{\mathfrak{p}}\right)}\right)=\tau\left(\frac{a_{\mathfrak{p}}^{2}}{\epsilon\left(\text { Frob }_{\mathfrak{p}}\right)}\right)$ for all $\mathfrak{p} \notin S$, by Cebotarev density theorem, we have

$$
\frac{\left(\operatorname{Trace} V_{\sigma}\right)^{2}}{\operatorname{det}\left(V_{\sigma}\right)}=\frac{\left(\operatorname{Trace} V_{\tau}\right)^{2}}{\operatorname{det}\left(V_{\tau}\right)}
$$

Restricting $V_{\sigma}$ and $V_{\tau}$ to $H$, we have $\operatorname{Trace}\left(\left.V_{\sigma}\right|_{H}\right)= \pm \operatorname{Trace}\left(\left.V_{\tau}\right|_{H}\right)$. We may choose $K^{\prime}$ even larger, so $H$ smaller, and get an equality $\operatorname{Trace}\left(\left.V_{\sigma}\right|_{H}\right)=$ Trace $\left(\left.V_{\tau}\right|_{H}\right)$. (See page 324 of [11].) Therefore $V_{\sigma} \cong V_{\tau}$ as $\mathbb{Q}_{l}[H]$-modules.

We construct a map $\alpha: G_{K} \rightarrow E^{\times} / F^{\times}$as follows. For any element $g \in G_{K}$ and $\phi \in \operatorname{End}_{\overline{\mathbb{Q}}}(M) \otimes_{\mathbb{Z}} \mathbb{Q}, \phi$ and $g(\phi)$ are inner by Skolem-Noether Theorem. So there exists an element $\alpha(g) \in \operatorname{End}_{\overline{\mathbb{Q}}}(M) \otimes_{\mathbb{Z}} \mathbb{Q}$, such that $g(\phi)=\alpha(g) \circ \phi \circ \alpha(g)^{-1}$. Since $E=\operatorname{End}_{K}(M) \otimes_{\mathbb{Z}} \mathbb{Q}$ is its own centralizer in $\operatorname{End}_{\overline{\mathbb{Q}}}(M) \otimes_{\mathbb{Z}} \mathbb{Q}$, we must have $\alpha(g) \in E$ and $\alpha(g)$ is determined up to a scalar multiple of $F^{\times}$. We have the following proposition.

Proposition 4.1. With the notation as above, we have $\alpha^{2} \equiv \epsilon\left(\bmod F^{\times}\right)$. Moreover, suppose that $\mathfrak{p}$ is a prime of good reduction for $M$ such that $a_{\mathfrak{p}} \neq 0$, then $\alpha\left(\right.$ Frob $\left._{\mathfrak{p}}\right) \equiv a_{\mathfrak{p}}\left(\bmod F^{\times}\right)$.

Proof. Fix a prime number $l$ which splits completely in $E$. To prove that $\alpha^{2} \equiv$ $\epsilon\left(\bmod F^{\times}\right)$, it suffices to prove that $\sigma\left(\frac{\alpha^{2}(g)}{\epsilon(g)}\right)=\tau\left(\frac{\alpha^{2}(g)}{\epsilon(g)}\right)$ for any $\sigma$ and $\tau$ are embeddings $E \hookrightarrow \mathbb{Q}_{l}$ which agree on $F$. Let $\sigma$ and $\tau$ be embeddings $E \hookrightarrow \mathbb{Q}_{l}$ which agree on $F$, then from the proof of the above theorem, we have $V_{\sigma} \cong V_{\tau} \otimes \psi$ for some character $\psi: G_{K} \rightarrow \mathbb{Q}_{l}^{\times}$. Consider the $H_{o m_{\mathbb{Q}_{l}}}\left(V_{\sigma}, V_{\tau}\right)$ as a Galois module. On one hand, $G_{K}$ acts on it by multiplication $\psi(g)^{-1}$. On the other hand, $G_{K}$ acts on it by conjugation by $\alpha(g)$. Note that $\alpha(g)$ acts on $V_{\sigma}$ and $V_{\tau}$ by $\sigma(\alpha(g))$ and $\tau(\alpha(g))$ respectively, $\alpha(g)$ acts on $H_{o m} \mathbb{Q}_{l}\left(V_{\sigma}, V_{\tau}\right)$ as $\tau(\alpha(g)) / \sigma(\alpha(g))$. Therefore, $\psi(g)^{-1}=\tau(\alpha(g)) / \sigma(\alpha(g))$ as elements in $\mathbb{Q}_{l}$. From the proof of the above theorem, we have $\epsilon^{\sigma}=\psi^{2} \cdot \epsilon^{\tau}$. It is easy to see that $\sigma\left(\frac{\alpha^{2}(g)}{\epsilon(g)}\right)=\tau\left(\frac{\alpha^{2}(g)}{\epsilon(g)}\right)$.

From the above argument, we have the following two equations: (1) $\sigma\left(a_{\mathfrak{p}}\right)=$ $\tau\left(a_{\mathfrak{p}}\right) \psi\left(\right.$ Frob $\left._{\mathfrak{p}}\right)$ because $V_{\sigma} \cong V_{\tau} \otimes \psi ;(2) \psi(g)^{-1}=\tau(\alpha(g)) / \sigma(\alpha(g))$ proved above. Therefore, we have $\sigma\left(\frac{\alpha\left(\text { Frob }_{\mathfrak{p}}\right)}{a_{\mathfrak{p}}}\right)=\tau\left(\frac{\alpha\left(\text { Frob }_{\mathfrak{p}}\right)}{a_{\mathfrak{p}}}\right)$. The result follows.

\subsection{Modularity}

The following two propositions generalize Proposition 5.4 and 7.1 of [12], where the statements are about the case $K=\mathbb{Q}$. The proof is the similar. We give details here for completeness. 
Proposition 4.2. Under Tate conjecture, the Galois representation $\rho_{\lambda}: G_{K} \rightarrow$ $G L_{2}\left(E_{\lambda}\right)$ is absolutely irreducible for all $\lambda$. In particular, we have $\operatorname{End}_{G_{K}}\left(\rho_{\lambda}\right)=$ $E_{\lambda}$.

Proof. By Tate conjecture, we have the following equality

$$
\prod_{\lambda \mid l} E_{\lambda}=E \otimes_{\mathbb{Q}} \mathbb{Q}_{l}=\operatorname{End}_{\mathbb{Q}_{l}\left[G_{K}\right]}\left(V_{l}\right)=\prod_{\lambda \mid l} \operatorname{End}_{\mathbb{Q}_{l}\left[G_{K}\right]}\left(V_{\lambda}\right) .
$$

We must have $E_{\lambda}=\operatorname{End}_{\mathbb{Q}_{l}\left[G_{K}\right]}\left(V_{\lambda}\right)$. It is obvious that

$$
\operatorname{End}_{E_{\lambda}\left[G_{K}\right]}\left(V_{\lambda}\right) \subset \operatorname{End}_{\mathbb{Q}_{l}\left[G_{K}\right]}\left(V_{\lambda}\right),
$$

therefore $\operatorname{End}_{E_{\lambda}\left[G_{K}\right]}\left(V_{\lambda}\right)=E_{\lambda}$.

Proposition 4.3. Under Tate conjecture, $\bar{\rho}_{\lambda}$ is absolutely irreducible for all but finitely many $\lambda$.

Proof. Since $E=\operatorname{End}_{K}(M) \otimes \mathbb{Q}$, we may assume that $\operatorname{End}_{K}(M)=\mathcal{O}_{E}$, the ring of integers of $E$. By Tate conjecture, the subalgebra $\mathbb{F}_{l}\left[\bar{\rho}_{l}\left(G_{K}\right)\right]$ of $\operatorname{End}_{\mathbb{F}_{l}}\left(H_{e t}\left(M, \mathbb{Z}_{l}\right) / l H_{e t}\left(M, \mathbb{Z}_{l}\right)\right)$ is a semi-simple $\mathbb{F}_{l}$-algebra. Let $\Phi: \operatorname{End}_{K}(M) \otimes$ $\mathbb{Z} / \mathbb{Z}_{l} \rightarrow \operatorname{End}_{\mathbb{F}_{l}}\left(H_{e t}\left(M, \mathbb{Z}_{l}\right) / l H_{e t}\left(M, \mathbb{Z}_{l}\right)\right)$ be the natural map. Then by bicommutation theorem, we have

$$
\operatorname{End}_{\mathbb{F}_{l}\left[G_{K}\right]}\left(H_{e t}\left(M, \mathbb{Z}_{l}\right) / l H_{e t}\left(M, \mathbb{Z}_{l}\right)\right)=\left(\mathbb{F}_{l}\left[\bar{\rho}_{l}\left(G_{K}\right)\right]\right)^{\circ} \operatorname{Im}(\Phi),
$$

where $\circ$ means commutator. Therefore, we have the following surjection map

$$
\operatorname{End}_{K}(M) \otimes \mathbb{Z} / \mathbb{Z}_{l}=\mathcal{O}_{E} \otimes \mathbb{Z} / \mathbb{Z}_{l} \rightarrow \operatorname{End}_{\mathbb{F}_{l}\left[G_{K}\right]}\left(H_{e t}\left(M, \mathbb{Z}_{l}\right) / l H_{e t}\left(M, \mathbb{Z}_{l}\right)\right) .
$$

By Tate conjecture again, the source and target have the same rank over $\mathbb{F}_{l}$. Therefore, the map is actually bijective. Let $k_{\lambda}$ be the residue field of $\mathcal{O}_{E_{\lambda}}$, then we have

$$
k_{\lambda}=\operatorname{End}_{k_{\lambda}\left[G_{K}\right]}\left(H_{e t}\left(M, \mathbb{Z}_{l}\right) / l H_{e t}\left(M, \mathbb{Z}_{l}\right)\right) .
$$

The statement follows.

In [1], the authors made the following conjecture.

Conjecture 4.4 (Serre's conjecture). Let $K$ be a totally real field. If $\bar{\rho}: G_{K} \rightarrow$ $G L_{2}\left(\overline{\mathbb{F}}_{p}\right)$ is a continuous, irreducible, and totally odd Galois representation, then $\bar{\rho}$ is modular in the sense that $\bar{\rho} \cong \bar{\rho}_{f}$, where $\bar{\rho}_{f}$ is the modular Galois representation attached to a Hilbert modular eigenform.

We immediately have that the following corollary.

Corollary 4.5. Under Tate conjecture and Serre's conjecture, the Galois representation $\bar{\rho}_{\lambda}$ attached to a motive $M$ of $G L_{2}$-type is modular. 


\section{References}

[1] K. Buzzard, F. Diamond, F. Jarvis, On Serre's conjecture for mod $\ell$ Galois representations over totally real fields, Duke Math Journal 55 (2010), no. 1, $105-161$.

[2] P. Deligne, Valeurs de fonctions $L$ et périodes d'intégrales, Proceedings of Symposia in Pure Mathematics 33 (1979), no. 2, 313-346.

[3] P. Deligne, J. Milne, Hodge cycles on abelian varieties, Hodge cycles, Motives, and Shimura varieties, Lecture Notes in Math. Vol. 900, 1982.

[4] P. Deligne, J. Milne, Tanakian catogories, Hodge cycles, Motives, and Shimura varieties, Lecture Notes in Math. Vol 900, 1982.

[5] H. Hida, On the search of genuine p-adic modular L-functions for $G L(n)$, Monograph, Memoires SMF 67, 1996.

[6] A. Panchishkin, Motives for absolute Hodge cycles, in Motives vol 1, Amer. Math. Soc., Providence, RI, 1994, 461-483.

[7] K. Ribet, Abelian varieties over $\mathbb{Q}$ and modular forms, Algebra and topology 1992, 53-79, Korea Adv. Inst. Sci. Tech., Taejŏn, 1992.

[8] K. Ribet, Fields of definition of abelian varieties with real multiplication, Arithmetic geometry (Tempe, AZ, 1993), 107-118, Contemp. Math. 174, Amer. Math. Soc., Providence, RI, 1994.

[9] A.J. Scholl, Motives for modular forms, Inventiones Mathematicae 100, no. $1,419-430$.

[10] J.-P. Serre, Modular forms of weight one and Galois representations, in Algebraic number fields: L-functions and Galois properties. Proc. Sympos., Univ. Durham, Durham, 1975, Academic Press, London, 1977, 193-268.

[11] J.-P. Serre, Propriétés galoisiennes des points d'ordre fini des courbes elliptiques, Invent. Math. 15 (1972), 259-331

[12] T. Yamauchi, $\mathbb{Q}$-motives and modular forms, Journal of Number Theory 128 (2008), 1485-1505.

Address: Chuangxun Cheng: Department of Mathematics, Bielefeld University, Germany.

E-mail: ccheng@math.uni-bielefeld.de

Received: 7 December 2012 
